\title{
Periodic Series of Peaks in Diffraction Patterns of Aperiodic Structures
}

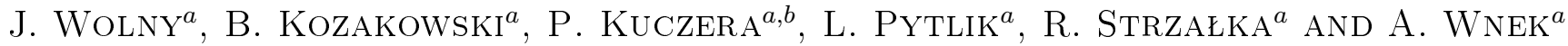 \\ ${ }^{a}$ Faculty of Physics and Applied Computer Science, AGH University of Science and Technology, Krakow, Poland \\ ${ }^{b}$ Laboratory of Crystallography, ETH Zurich, Switzerland
}

\begin{abstract}
Quasicrystals are aperiodic structures with no periodicity both in direct and reciprocal space. The diffraction pattern of quasicrystals consists however of the periodic series of peaks in the scattering vector space. The intensities of the peaks of all series reduced in a proper way build up the so-called envelope function common for the whole pattern. The Fourier transformed envelope gives the average unit cell which is the statistical distribution of atomic positions in physical space. The distributions lifted to high dimensions correspond to atomic surfaces - the basic concept of structural quasicrystals modeling within higher-dimensional approach.
\end{abstract}

DOI: $10.12693 /$ APhysPolA.126.625

PACS: 61.44.-n

\section{Introduction}

Quasicrystals were introduced to physics and materials science in 1984 [1, 2], two years after Shechtman's discovery. One of the most distinguishing features of quasicrystals is their diffraction pattern. The structure of quasicrystals is aperiodic; however their diffraction patterns contain sharp peaks, which is the proof of their perfect long-range order. Because of their incompatibility with translational long-range order observed for periodic crystals, the patterns exhibit point symmetries forbidden in classical crystallography (e.g. 5-fold). The reverse reasoning, that is that the structure aperiodicity implies pattern aperiodicity is not true. We show that the periodicities in the diffraction patterns of aperiodic structures yield important information about their structure. In this paper three structure models will be analyzed: a 1D quasicrystal - the Fibonacci chain, a 2D quasicrystal represented by the rhombic Penrose tiling, and an incommensurately modulated structure with harmonic modulation.

\section{Diffraction pattern of the Fibonacci chain}

An example of a 1D quasicrystal is represented by the Fibonacci chain of two segments $L=\tau$ and $S=1$. Its diffraction pattern (Fig. 1a) contains only the Bragg peaks arranged aperiodically. However the aperiodic pattern is a superposition of an infinite number of periodic series of peaks [3]. Three such series, marked by $m=0,12$, are highlighted in Fig. 1a. Each series has the same periodicity (here $k_{0}=2 \pi / a_{0} \approx 4.547$, where $a_{0}=1 / \tau^{2} \approx 1.382$ is an average distance between points in the chain, $\tau \approx 1.618$ is the golden mean). The shift between two series is constant and equal to $k_{1}=k_{0} \sqrt{5}=2 \pi \tau \approx 10.17$. Every peak position $k$ can be reached by using only two periods: $k_{1}$ and $k_{0}$. An interesting feature of the pattern appears when we subtract the $k_{1}$ component from the coordinates of the peaks. The peak maxima form a curve called "envelope" (Fig. 1b).

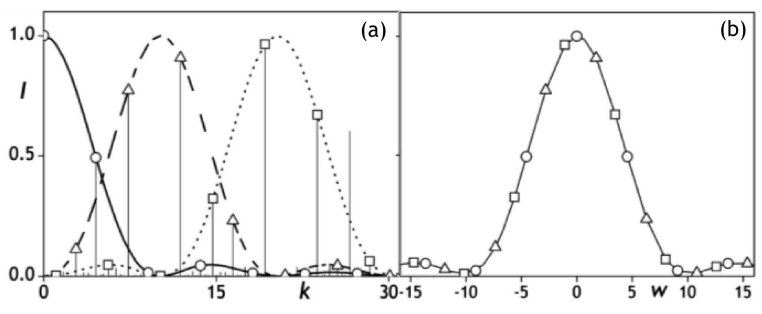

Fig. 1. Diffraction pattern of the Fibonacci chain. (a) Three infinite periodic series of peaks. (b) The envelope function running over the maxima of all peaks is the result of reducing peak positions by: $w=k-m k_{1}$. Three different symbols of points correspond to three series of peaks from part (a).

\section{Average unit cell for the Fibonacci chain}

The envelope curve is the square of a Fourier transform of an average unit cell (AUC). The AUC is a distribution $P(u)$ of reduced atomic positions $u=x \bmod \left(a_{0}\right)$, where $x$ is the atomic position in physical space (Fig. 2a). Conversely, we can determine the AUC directly from the diffraction pattern and its envelope curve, and obtain the atomic positions. Due to the phase recovery problem this is not straightforward. For a centrosymmetric structure, the phase takes only two values: 0 or $\pi$. The phase changes its value every time the envelope function from Fig. $1 \mathrm{~b}$ reaches zero. This simple rule allows us to obtain the distribution of atomic positions (Fig. 2a) without the necessity to fit the phase values iteratively.

\section{TAU2-scaling}

Usually the modulation wave vector $q$ is used to index peak positions: $q=k / \tau[4-6]$.

To reconstruct the whole pattern the $P(u, v)$ distribution has to be determined where $v$ is a reduced position with respect to the reference frame with period of $a_{0} \tau$ [4-6]. This distribution is non-zero only along a certain line (Fig. 2b). After a proper shift (dashed line in 
Fig. $2 \mathrm{~b}$ ) the equation of the line is $v=-\tau^{2} u+b$. The factor $-\tau^{2}$ is characteristic for all decagonal and icosahedral quasicrystals; (this is called TAU2-scaling). It gives an immediate result for the peak's phase $(0$ or $\pi): \eta q_{0} b$ (where $\eta$ is an integer) - on condition that $P(u, v)$ is shifted to the points of symmetry of the reference frame.

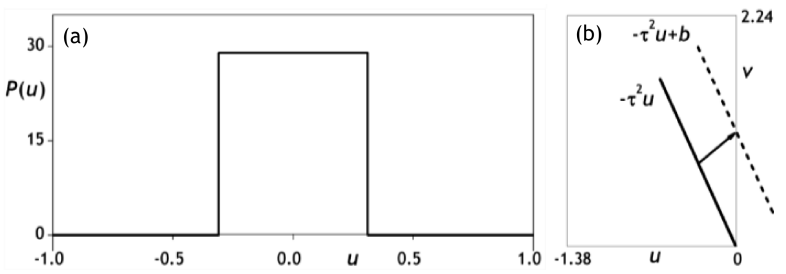

Fig. 2. (a) The AUC for a Fibonacci chain obtained as a product of the inverse Fourier transform of the envelope function of the structure factor (theoretical shape). (b) The AUC is non-zero only along a line segment.

\section{Rhombic Penrose tiling}

The rhombic Penrose tiling is a paradigmatic example of a $2 \mathrm{D}$ quasiperiodic structure. To index all peaks in the diffraction pattern one needs to choose two $2 \mathrm{D}$ primary

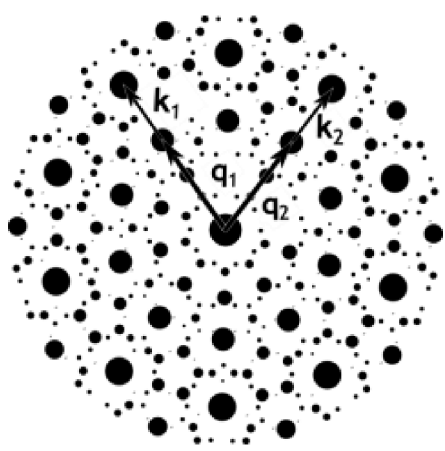

Fig. 3. Diffraction pattern of the rhombic Penrose tiling. The basis vectors $\boldsymbol{k}_{1}, \boldsymbol{k}_{2}, \boldsymbol{q}_{1}, \boldsymbol{q}_{2}$ used for the construction of the AUC are shown.
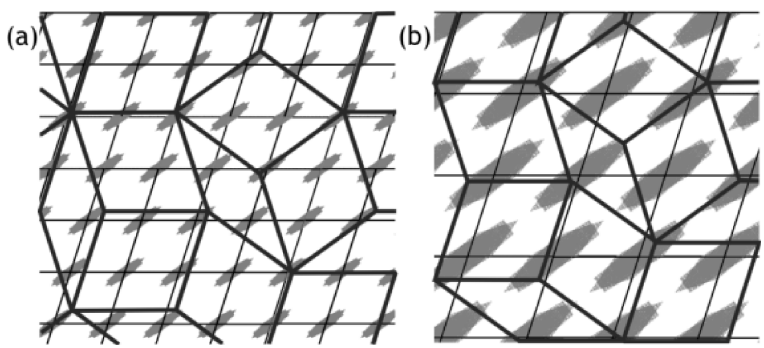

Fig. 4. Expanded AUC of the rhombic Penrose tiling constructed with: (a) basis vectors $\boldsymbol{k}_{1} \approx(0,30.77), \boldsymbol{k}_{2} \approx$ $(31.16,10.13)$ and $(\mathrm{b})$ basis modulation vectors. Set of basis vectors indicated in Fig. 3. Thick lines correspond to the rhombic Penrose tiling quasilattices, thin lines to the lattice related to the AUC. Overlapping pentagons in gray. wave vectors $\boldsymbol{k}_{1}$ and $\boldsymbol{k}_{2}$, and two $2 \mathrm{D}$ modulation vectors $\boldsymbol{q}_{1}$ and $\boldsymbol{q}_{2}: \boldsymbol{q}_{i}=\boldsymbol{k}_{i} / \tau$ (Fig. 3). The peak position is an integer linear combination of these four basis vectors. The diffraction pattern is a set of strictly periodic series of diffraction peaks. The expanded AUC for the rhombic Penrose tiling is shown in Fig. 4. A single AUC consists of four overlapping pentagons (two large ones and two small ones) which are the equivalents of pentagonal atomic surfaces in perpendicular space [7].

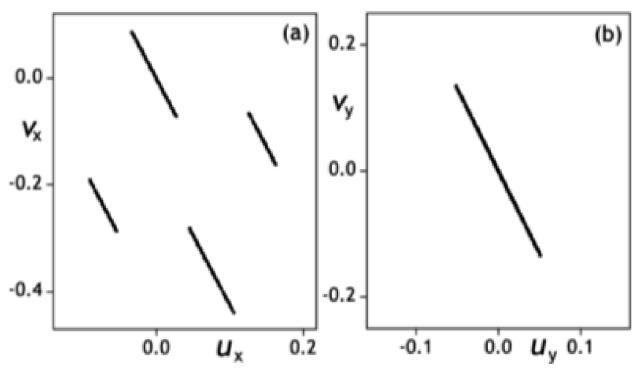

Fig. 5. TAU2-scaling of AUCs of the rhombic Penrose tiling shown in Fig. 4 . (a) Scaling relation for $\left(u_{x}, v_{x}\right)$ and (b) $\left(u_{y}, v_{y}\right)$-coordinates.

\section{1D incommensurately modulated structure}

Yet another example of a modulated structure is a onedimensional periodic structure with an incommensurate harmonic modulation. The position of an atom in such a structure is given by: $x_{n}=n a+A \sin \left(q_{0} n a\right)$, with $a$ being the base period, $A$ the modulation amplitude, $q_{0}$ the modulation vector (incommensurate with respect to the base period), $n$ an index numbering the atoms. The diffraction pattern consists of a periodic series of diffraction peaks and satellite peaks (Fig. 6a). The positions

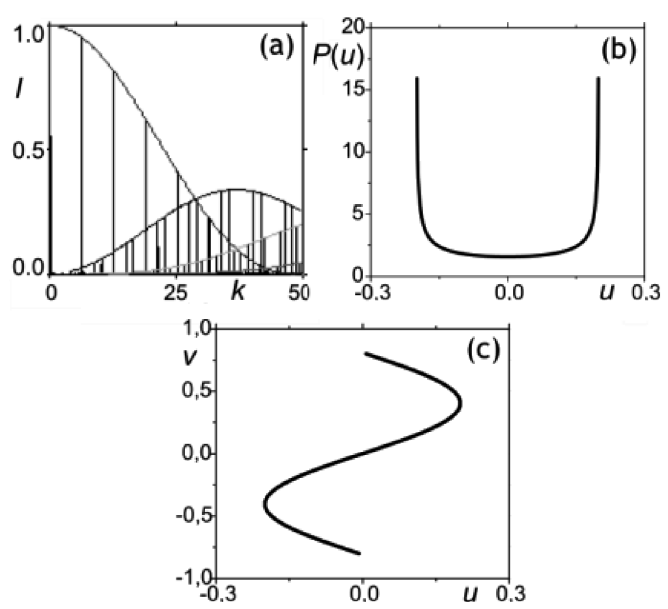

Fig. 6. (a) Diffraction pattern of a 1D incommensurately modulated structure. The envelopes of the main peaks and a few nearest satellites are with marked. (b) AUC of a 1D harmonically modulated structure. (c) Correlation trace of AUCs derived for two wave vectors: $k_{0}, q_{0}=k / \tau$ for a structure with incommensurate harmonic modulation. 
of periodic and satellite peaks are incommensurate in relation to each other. Figure $6 \mathrm{~b}$ demonstrates this relationship along with the appropriate envelope functions. The observed periodicity allows us to construct an AUC for the wave vector $k_{0}=2 \pi / a$ and the incommensurate wave vector $q_{0}$ [8]. The correlation between these two vectors is shown in Fig. 6c. It is not linear as distinct from quasicrystals.

\section{Periodicities of the diffraction pattern}

In Fig. 7 the idea of higher-dimensional description (the cut-and-project method) is schematically shown. The nodes of a $2 \mathrm{D}$ periodic square lattice (with parameter $A$ ) restrained to the projection strip are projected onto the physical-space direction $x_{\|}$.

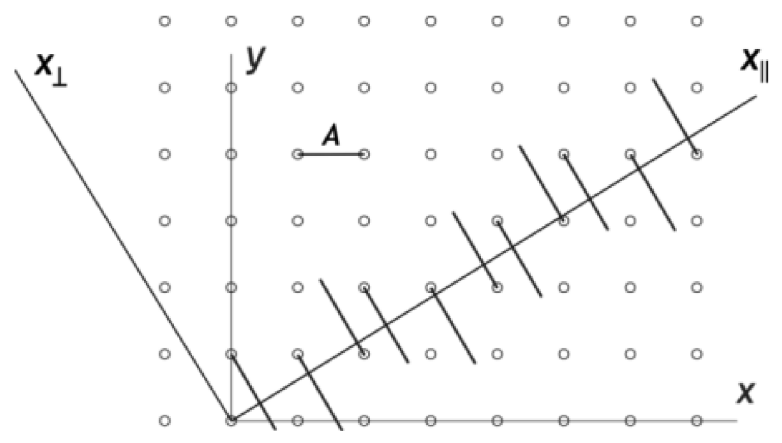

Fig. 7. The Fibonacci chain represented as a projection of $2 \mathrm{D}$ square direct space lattice onto the physical space $\left(x_{\|}\right)$. The segments in red are the atomic surfaces known from cut-and-project method and correspond to line segments from Fig. 2a called AUC.

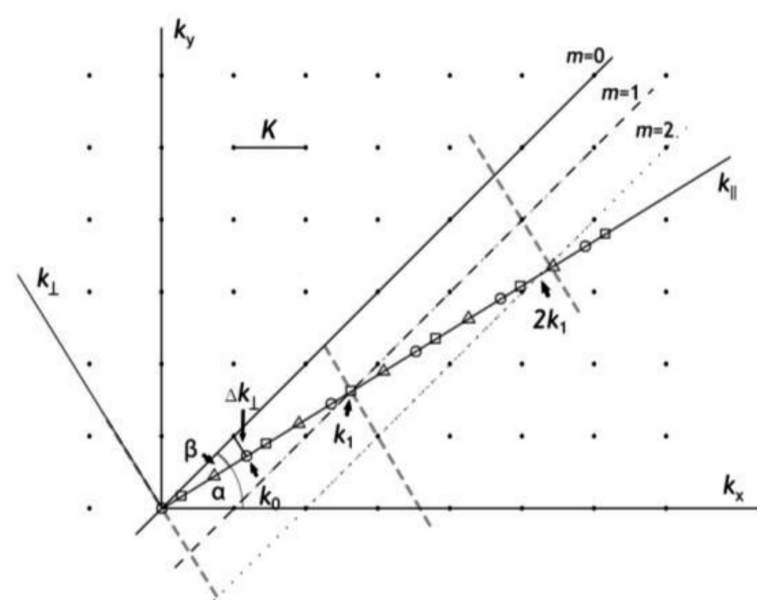

Fig. 8. The diffraction pattern of the Fibonacci chain represented as a projection of the $2 \mathrm{D}$ square reciprocal lattice onto physical space $\left(k_{\|}\right)$. Different symbols of points correspond to three series of peaks from Fig. 1a.

To fully understand the existence of the two periodicities in the diffraction pattern we now introduce a $2 \mathrm{D}$ space description. Let us assume a $2 \mathrm{D}$ square reciprocal space lattice with lattice constant $K=\frac{2 \pi}{A}=\frac{2 \pi}{\sqrt{\tau^{2}+1}} \approx$ 3.30 (Fig. 8). The $k_{1}$ periodicity implies an infinite set of parallel and equidistant lines (marked with solid, dashed and dotted lines). Here the $(1,1)$ direction in $2 \mathrm{D}$ reciprocal space was chosen. From Fig. 8 one can explicitly infer the value of the $k_{1}$ period, since

$$
\begin{aligned}
& \left\{\begin{array} { l } 
{ k _ { 1 x } = \tau k _ { 1 y } } \\
{ k _ { 1 y } = k _ { 1 x } - K }
\end{array} \rightarrow \left\{\begin{array}{l}
k_{1 x}=\tau^{2} K \\
k_{1 y}=\tau K
\end{array}\right.\right. \\
& \rightarrow k_{1}=\sqrt{k_{1 x}^{2}+k_{1 y}^{2}}=2 \pi \tau \approx 10.17
\end{aligned}
$$

The $k_{0}$ periodicity is an infinite periodic set of points (open circles along each of the lines). If the inclination angle $\alpha$ of the lines related to periodicity $k_{1}$ is properly chosen $\left(\alpha=31.72^{\circ}\right.$ such as $\left.\tan (\alpha)=1 / \tau\right)$, the points related to the periodicity $k_{0}$ will form a square lattice. Peak positions (marked with different symbols) are obtained by projecting the periodic set of points from each line onto the physical space $\left(k_{\|}\right)$. The perpendicular space is spanned along the projection direction $\left(k_{\perp}\right)$. The trace of the envelope function from Fig. 1b is placed in the perpendicular space (marked in gray segment dashed lines in Fig. 8). To be more precise, the structure factor should be considered rather than the intensity. The envelopes are rescaled according to the formula: $k_{\perp}=w / \tau^{3}$, where $w=k_{\|} \bmod \left(k_{1}\right)$; they are centered at each of the parallel lines related to the periodicity $k_{1}$. The scaling factor in reciprocal space is $1 / \tau^{3}$; it is the same for both $k_{\|}$and $k_{\perp}$. This clearly follows from Fig. 8: $\Delta k_{\perp} / k_{0}=\tan \beta=\tan \left(45^{\circ}-\alpha\right)=1 / \tau^{3}$. The intensity of a given peak is defined by the values of the respective envelope function at $k_{\perp}=0$.

\section{Correspondence to the high-dimensional approach}

Two characteristic features of the diffraction pattern of quasicrystals can be easily explained by the construction in Fig. 8: (1) pattern is infinitely dense because the set of lines related to the periodicity $k_{1}$ is infinite; (2) the intensities of the peaks with higher $k_{\perp}$ component are weaker because the envelope function decays asymptotically.

After transformation to direct space, the square reciprocal lattice transforms into a square lattice and the Fourier transform of the envelope function of the structure factor in the perpendicular space produces an atomic surface (also-called by other names, such as "acceptance domain" or simply "window"). Such an approach, based on construction of a square lattice, is the foundation of the higher-dimensional approach [9-14].

\section{Summary}

A diffraction pattern can be divided into periodic series of peaks; this is true for periodic crystals, quasicrystals and modulated structures (with commensurate or incommensurate modulation). This approach applies to aperiodic structures like the Thue-Morse sequence as well [15]. 
We use here the periodicity in the wave vector space (reciprocal space) to construct a reference frame in the physical space. The positions of atoms within a model structure reduced to one period of a reference frame form the average unit cell. If the ratio of periodicities of two AUCs is $\tau$ (the ratio of the wave vectors, for which the AUCs were calculated, is $1 / \tau)$, then they are related to each other by the TAU2-scaling. The TAU2-scaling is a linear relationship between the non-zero density regions of the two AUCs with the linear coefficient equal to $\left(-\tau^{2}\right)$. This scaling is representative for all commonly observed quasicrystals and enables us to use it to derive the structure factor for decagonal quasicrystals (if their structure can be described as a decorated rhombic Penrose tiling) and subsequently refine a number of quasicrystalline structures [16].

The occurrence of the periodic series in diffraction pattern holds for any structure and not only quasicrystals. A series of peaks can be complemented by an envelope being a Fourier transform of an AUC. The properties of the envelope make it possible to determine the phases of the peaks. As this approach is general and well-defined in a mathematical sense it can be used in the refinement of more complex structures like proteins, for instance. The approach works not only for the Bragg peaks but also for the singular continuous component (which scales in a fractal way with the number of scattering atoms) as well as for the absolutely continuous component. From an experimental point of view the latter is diffuse scattering (cf. [17]). Using this approach the full structural knowledge can be obtained from the diffraction pattern and not only from the position and intensity of the peaks.

\section{Acknowledgments}

We acknowledge partial suport by Polish National Science Centre (NCN) under grant No. DEC2013/11/B/ST3/03787. R.S. has been partly supported by the EU Human Capital Operation Program, Polish Project No. POKL.04.0101-00-434/08-00.

\section{References}

[1] D. Shechtman, I. Blech, D. Gratias, J.W. Cahn, Phys. Rev. Lett. 53, 1951 (1984).

[2] D. Levine, P.J. Steinhardt, Phys. Rev. Lett. 53, 2477 (1984).

[3] J. Wolny , B. Kozakowski, P. Kuczera, R. Strzałka, A. Wnek, Isr. J. Chem. 51, 1275 (2011).

[4] P. Buczek, L. Sadun, J. Wolny, Acta Phys. Pol. B 36, 919 (2005).

[5] J. Wolny, Philos. Mag. A 77, 395 (1998).

[6] J. Wolny, Acta Crystallogr. A 54, 1014 (1998).

[7] W. Steurer, T. Haibach, Acta Crystallogr. A 55, 48 (1999).

[8] G. Urban, J. Wolny, Philos. Mag. 86, 629 (2006).

[9] N.G de Bruijn, Nederl. Akad. Wetensch. Proc. Ser. A 84, 38 (1981).

[10] P. Kramer, R. Neri, Acta Crystallogr. A 40, 580 (1984).

[11] M. Duneau, A. Katz, Phys. Rev. Lett. 54, 2688 (1985).

[12] P.A. Kalugin, A.Yu. Kitaev, L.S. Levitov, J. Physique Lett. 46, L601 (1985).

[13] A. Cervellino, W. Steurer, Acta Crystallogr. A 58, 180 (2002).

[14] H. Takakura, A. Yamamoto, A.P. Tsai, Acta Crystallogr. A 57, 576 (2001).

[15] J. Wolny, A. Wnek, J.L. Verger-Gaugry, J. Comput. Phys. 163, 313 (2000).

[16] P. Kuczera, J. Wolny, W. Steurer, Acta Crystallogr. $B$ 68, 578 (2012).

[17] D. Orzechowski, J. Wolny, Philos. Mag. 87, 3049 (2007). 